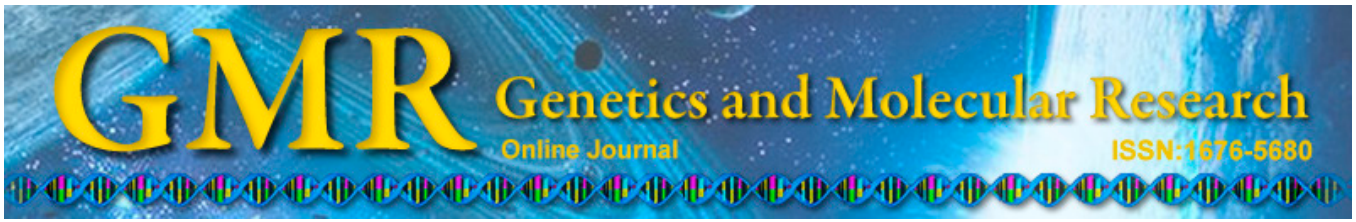

\title{
Identification of heat shock proteins via transcriptome profiling of tree peony leaf exposed to high temperature
}

\author{
Y.Z. Zhang*, Y.W. Cheng*, H.Y. Ya*, J.M. Han and L. Zheng \\ Life Science Department, Luoyang Normal University, Luoyang, China \\ *These authors contributed equally to this study. \\ Corresponding author: Y.W. Cheng \\ E-mail: ywei_cheng@163.com
}

Genet. Mol. Res. 14 (3): 8431-8442 (2015)

Received November 24, 2014

Accepted March 26, 2015

Published July 28, 2015

DOI http://dx.doi.org/10.4238/2015.July.28.10

\begin{abstract}
The tree peony leaf is an important vegetative organ that is sensitive to abiotic stress and particularly to high temperature. This sensitivity affects plant growth and restricts tree peony distribution. However, the transcriptomic information currently available on the peony leaf in public databases is limited. In this study, we sequenced the transcriptomes of peony leaves subjected to high temperature using the Illumina HiSeq TM 2000 platform. We performed de novo assembly of 93,714 unigenes (average length of $639.7 \mathrm{bp}$ ). By searching the public databases, 22,323 unigenes and 13,107 unigenes showed significant similarities with proteins in the NCBI non-redundant protein database and SWISS-PROT database (E-value $<1 \mathrm{e}-5$ ), respectively. We assigned 17,340 unigenes to Gene Ontology categories, and we assigned 7618 unigenes to clusters of orthologous groups for eukaryotic complete genomes. By searching the Kyoto Encyclopedia of Genes and Genomes Pathway database, 8014 unigenes were assigned to 6 main categories, including 290 KEGG pathways. To advance research on improving thermotolerance, we identified 24 potential heat shock protein genes with complete open reading frames from the transcriptomic sequences.
\end{abstract}


This is the first study to characterize the leaf transcriptome of tree peony leaf using high-throughput sequencing. The information obtained from the tree peony leaf is valuable for gene discovery, and the identified heat shock protein genes can be used to improve plant stress-tolerance.

Key words: Heat shock protein; High temperature; Transcriptome; Tree peony leaf

\section{INTRODUCTION}

A tree peony (Paeonia suffruticosa) is a type of woody shrub that belongs to the Paeoniaceae family and produces extremely popular ornamental flowers. In China, the tree peony is known as "kings of flowers", and large-scale planting of tree peonies in cities including Luoyang (Henan Province) and Heze (Shandong Province) formed famous landscapes that bring many tourism benefits. Moreover, the peony root bark, also referred to as "Danpi" in Chinese, is an important Chinese medicine. The seed oil is a popular product for cooking because it is considered to be healthy. Tree peony thrives in warm and cold climates, with an optimum growth temperature of $16^{\circ}$ to $20^{\circ} \mathrm{C}$. In summer, high temperatures prevent plant growth and typically damage the leaves, which is a limiting factor for the growth distribution and plant production. However, transcriptome profiling of leaves under heat shock stress has not been thoroughly conducted, and the understanding of the thermotolerance mechanism is limited.

One of the most important thermotolerance mechanisms in plants is the induction of heat shock proteins (Hsps), which typically act as molecular chaperones to reduce damage to cells (Wang et al., 2004; Xue et al., 2014). Based on their molecular weights, plant Hsps are divided into 5 groups, including Hsp100, Hsp90, Hsp70, Hsp60, and the small Hsp (sHsp) family (Wang et al., 2004). In Arabidopsis, 8 Hsp100 members (Agarwal et al., 2001), 7 Hsp90 members (Krishna and Gloor, 2001), 18 Hsp70 members (Lin et al., 2001), 11 Hsp60 members (Hill and Hemmingsen, 2001), and 13 sHsps members (Scharf et al., 2001) have been identified in the genome and analyzed. Hsps from different families have been also reported in other plant species, such as cereals (Maestri et al., 2002), maize (Sun et al., 2012), and wheat (Kumar et al., 2012). Modifying the expression of $H_{s p}$ via genetic engineering has been shown to be useful for enhancing temperature stress tolerance in plants. For example, Arabidopsis and rice that overexpressed $H_{s p 101}$ showed superior tolerance to extreme temperatures (Queitsch et al., 2000; Katiyar-Agarwal et al., 2003). In tree peony, an Hsp 70 gene was identified by Li et al. (2011), but most Hsps remain unknown. Therefore, it is essential to identify additional Hsps for improving the stress tolerance of the tree peony.

Because of the lack of a reference genome, transcriptome sequencing is an optimal method for large-scale screening of Hsps in tree peony. Sequencing of plant transcriptomes has become feasible with the recent development of next-generation sequencing technology. For transcriptome sequencing in non-model plants, the Roche 454 platform was initially preferred because it generates long reads (Vera et al., 2008; Meyer et al., 2009). However, the short reads generated by the Illumina platform can currently be assembled for a lower price, and a series of transcriptomes from non-model organisms have been successfully sequenced and assembled de novo (Li et al., 2012; Toledo-Silva et al., 2013). For tree peony, transcriptomes of petals and flower buds have been sequenced and millions of transcripts were obtained (Zhang et al., 2013; Zhou et al., 2013). Therefore, the Illumina platform is a preferable method 
for the transcriptome sequencing of peony leaves. In the present study, we performed the de novo transcriptome sequencing of peony leaf using the Illumina HiSeq TM 2000 platform, and the unigenes were assembled and annotated. Furthermore, a series of abiotic stress-tolerant genes were identified. This study is the first attempt to characterize the leaf transcriptome in tree peony using high throughput sequencing. Our database of sequences will serve as an invaluable resource for novel gene discovery and molecular marker development for selective breeding.

\section{MATERIAL AND METHODS}

\section{Plant material and RNA extraction}

P. suffruticosa 'juanyehong' were planted at the Peony Institute of Luoyang (Luoyang, China). Leaves were collected from healthy plants at approximately $1300 \mathrm{~h}$ in July when the plants were experiencing temperatures of approximately $35^{\circ}$ to $37^{\circ} \mathrm{C}$. Two replicated samples were frozen immediately in liquid nitrogen and stored at $-80^{\circ} \mathrm{C}$. Total RNA was extracted using a cetyltrimethylammonium bromide-based protocol and further purified with the RNeasy Plant Mini Kit (Qiagen, Hilden, Germany). RNA quality was verified on a Bioanalyzer 2100 (Aligent, Santa Clara, CA, USA). RNA integrity number values were $>8.5$ for all samples.

\section{cDNA library construction and sequencing}

Poly (A)-containing RNA was collected from total RNA using magnetic oligo (dT) beads and was fragmented into small pieces using fragmentation buffer. Next, the short mRNA fragments were reverse-transcribed into first-strand cDNA using random hexamer primers, which was followed by second-strand cDNA synthesis. The double-stranded cDNA fragments were subjected to end repair, and a single nucleotide A (adenine) was added to the 3 ' ends. Sequencing adaptors were ligated to the 3 ' ends and the suitable fragments were enriched by polymerase chain reaction amplification. After validation with an Agilent 2100 Bioanalyzer, the cDNA library was sequenced using an Illumina HiSeq 2000 sequencing platform. The sequencing and preparation of libraries were performed by Encode Genomics Bio-Technology Co., Ltd. (Suzhou, China). Sequence data were deposited in the NCBI database under accession No. SRR1572176 and SRR1572177.

\section{De novo assembly and gene annotation}

The raw reads were cleaned by removing low-quality reads and ambiguous bases $(\mathrm{N})$ using a Perl script. De novo transcriptome assembly was performed using a paired-end method with the Trinity software (Grabherr et al., 2011). To remove redundancy among Trinity-generated transcripts, sequences were clustered using CD-HIT with thresholds of 0.95 (Fu et al., 2012). Based on annotation with the NR database, transcripts were further clustered by removing sequences of bacteria, viruses, and fungi. The remaining transcripts were designated as unigenes. For annotation, all assembled unigenes were searched against public databases, including NCBI non-redundant protein, SWISS-PROT, TrEMBL, and KOG databases using the BLAST program (E-value $<1 \mathrm{e}-5)$. Unigenes annotated in the TrEMBL and SWISS-PROT databases were listed and filtered to remove duplications. GO annotation of unigenes was performed using the Blast2GO software. Unigene sequences were also aligned to the Clusters of Ortholo- 
gous Group database to predict and classify functions. Pathway assignments were also carried out based on the KEGG database. Candidate Hsps were obtained by filtering out the result of $\mathrm{Nr}$ annotation, and open reading frames were identified using the ORF finder (http://www.ncbi. nlm.nih.gov/gorf/gorf.html). Subcellular localization of proteins was predicted using the online software Cell-PLoc 2.0 (http://www.csbio.sjtu.edu.cn/bioinf/Cell-PLoc-2/). Sequence alignment was performed using ClustalW and phylogenetic analysis was performed using MEGA 5 (Tamura et al., 2011) with the neighbor-joining method with 1000 bootstrap replicates.

\section{RESULTS}

\section{Illumina paired-end sequencing and de novo assembly}

Tree peony is typically subjected to extremely high temperature stress in summer. Two groups of leaves that were subjected to high temperatures were sampled in July. The cDNA libraries from the 2 RNA samples were constructed and sequenced separately using an Illumina HiSeq 2000 genome analyzer. Each sequenced sample can yield 2 x 100 bp of independent reads from either end of a DNA fragment. In this study, a total of 134,151 transcripts were assembled with an average length of 692.52 nucleotides and an N50 of 1217 nucleotides. After clustering of redundant transcripts, removing bacteria, viruses, and fungi sequences, the remaining 93,714 transcripts had an average length of $639.7 \mathrm{bp}$ and were designed as unigenes. The length distribution of unigenes is shown in Figure 1. Unigenes in the range of 200 to $500 \mathrm{bp}$ accounted for $63.4 \%$ of total unigenes. There were 14,836 unigenes ranging from 500 to $1000 \mathrm{bp}(15.8 \%), 10,194$ unigenes ranging from 1000 to $2000 \mathrm{bp}(10.9 \%)$, and 5542 unigenes with lengths of more than $2000 \mathrm{bp}(5.9 \%)$.

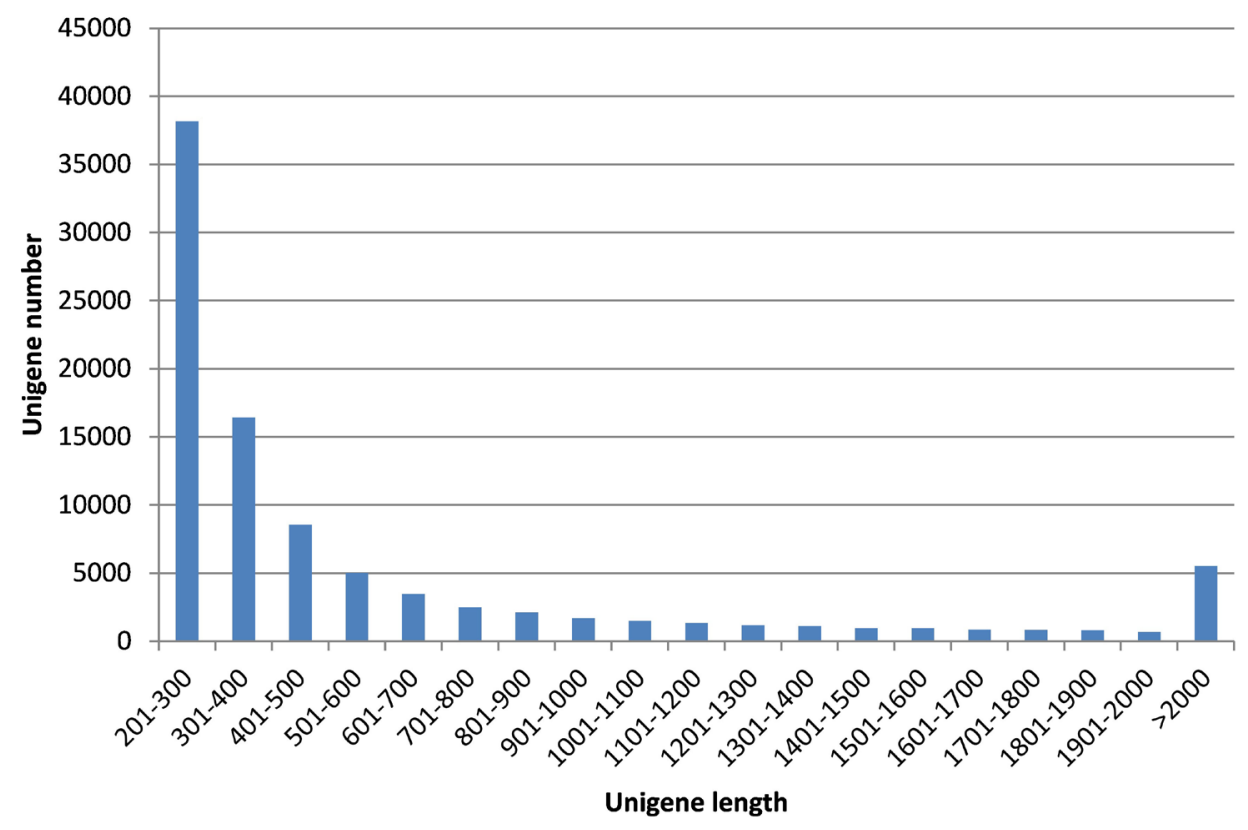

Figure 1. Assessment of the assembled quality of transcripts and unigenes by size distribution. 


\section{Functional annotation via public database search}

For validation and annotation of assembled unigenes, sequence-similarity searches were conducted by querying against the NCBI non-redundant protein $(\mathrm{Nr})$ database, SWISSPROT protein database, and TrEMBL database. The results showed that of 93,714 unigenes, 22,323 unigenes showed significant similarity to known proteins in the $\mathrm{Nr}$ database, 13,107 unigenes showed significant similarity in the SWISS-PROT database, and 22,680 unigenes showed significant similarity to known proteins in the TrEMBL database. Among the Nr hits, 12,696 unigenes $(56.9 \%)$ had significant matches with proteins from Vitis vinifera, which represented the largest group. We also analyzed the E-value and similarity distributions of unigenes matched in the $\mathrm{Nr}$ database. The results showed that $63.4 \%$ of the unigenes showed significant homology with E-values less than $1.0 \mathrm{e}-50$ and that $32.4 \%$ of the unigenes showed a high similarity with a value more than $80 \%$ (Figure $2 \mathrm{~A}$ and $\mathrm{C}$ ). For the BLAST results against the SWISS-PROT database, the corresponding proportions for E-value $(<1 \mathrm{e}-50)$ and similarity $(>80 \%)$ were 61.1 and $19.7 \%$, respectively (Figure $2 \mathrm{~B}$ and $\mathrm{D}$ ).
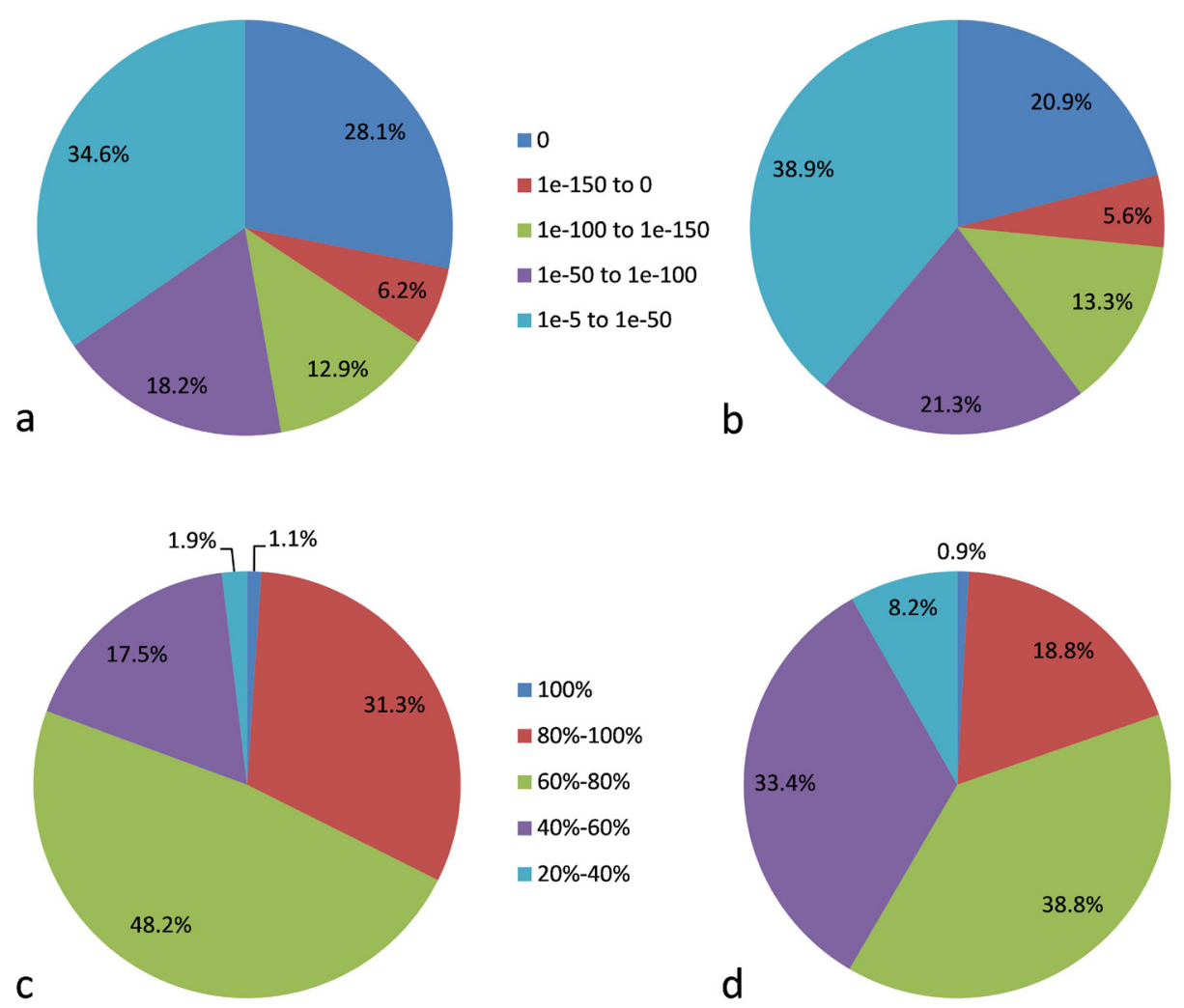

Figure 2. Characteristics of similarity search of unigenes against $\mathrm{Nr}$ and SWISS-PROT databases. A. E-value distribution of BLAST hits for the assembled unigenes with a cutoff of $1 \mathrm{e}-5$ in the $\mathrm{Nr}$ database. B. E-value distribution of BLAST hits for the assembled unigenes with a cutoff of $1 \mathrm{e}-5$ in the SWISS-PROT database. C. Similarity distribution of the top BLAST hits for the assembled unigenes with a cutoff of 1e-5 in the Nr database. D. Similarity distribution of the top BLAST hits for the assembled unigenes with a cutoff of 1e-5 in the SWISS-PROT database. 


\section{Functional classification by GO and KOG}

Based on the unigenes annotated in the SWISS-PROT and TrEMBL databases, 17,340 unigenes were assigned to 3 main GO categories, including molecular functions, biological processes, and cellular components (Figure 3). Among these, 14,292 unigenes were grouped in the category of biological processes, 8524 unigenes in cellular components, and 15,810 unigenes in molecular function. In the biological processes category, the items "metabolic process" (12,471 unigenes; $87.2 \%)$ and "cellular process" (10,577 unigenes; $74 \%)$ were prominently presented, indicating that important metabolic activities occur in the leaves of the peony tree. For the category of cellular components, "cell" (6545 unigenes; 76.8\%) and "cell part" (6544 unigenes; $76.8 \%$ ) were the most highly representative groups, followed by groups of "organelle" (4341 unigenes; 51\%) and "membrane" (3248 unigenes; 38.1\%). "Binding" (11,782 unigenes; $74.5 \%)$ and "catalytic activity" (9785 unigenes; 61.9\%) represented the largest number of groups in the molecular function category.

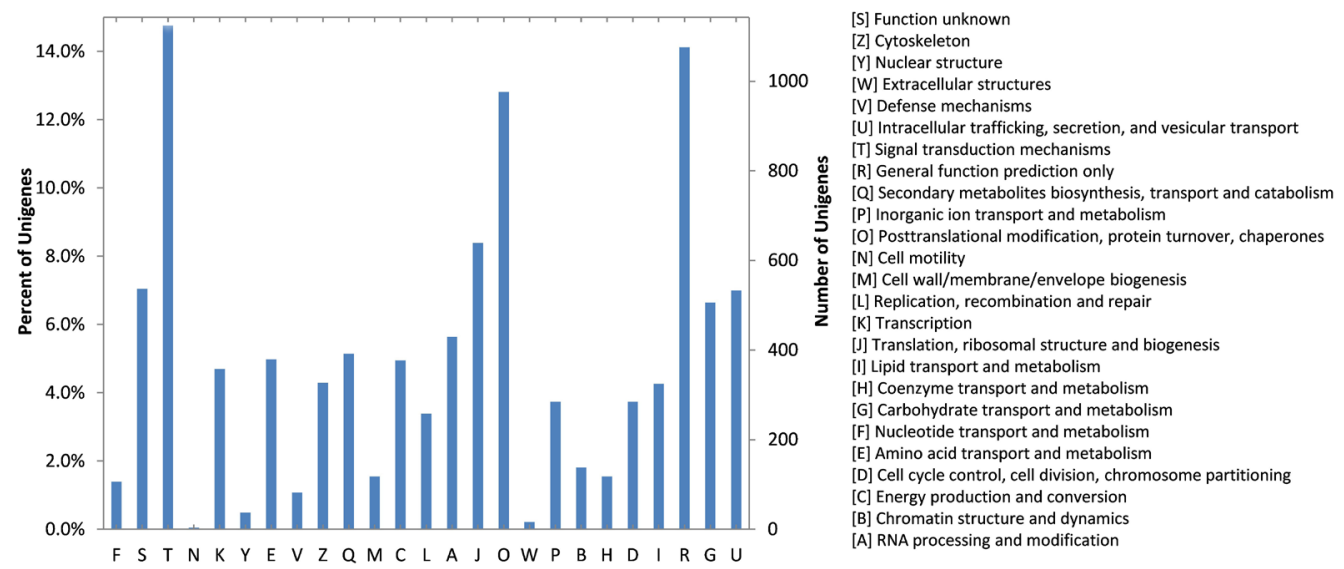

Figure 3. Gene ontology (GO) classification of assembled unigenes.

Unigenes annotated in the KOG databases were further classified, and 7618 unigenes were assigned to $25 \mathrm{KOG}$ categories (Figure 4). "Signal transduction mechanisms" and "general function prediction only" were the largest groups, accounting for 14.8 and $14.1 \%$ of unigenes annotated with KOG, respectively. The 3rd largest group was "posttranslational modification, protein turnover, chaperones" $(12.8 \%)$, whereas only a few unigenes were assigned to clusters of "extracellular structures" (16 unigenes) and "cell motility" (4 unigenes).

\section{Metabolic pathways}

Unigenes were used to search the KEGG database to further understand the biological functions and genes interactions. A total of 8014 unigenes were assigned to 6 main categories, including 290 KEGG pathways. Among the 6 main categories, "metabolism" contained the largest number of unigenes (3276) and accounted for $47.2 \%$ of the total (Figure 5A). The other 5 main categories were "genetic information processing" (2462 unigenes; 35.5\%), "human diseases" (1353 unigenes; 19.5\%), "organismal systems" (1019 unigenes; 14.7\%), "cel- 
lular processes" (975 unigenes; 14.1\%), and "environmental information processing" (640 unigenes; 9.2\%). In the metabolic pathway, unigenes were classified into 11 subcategories (Figure 5B), mainly including "amino acid metabolism", "biosynthesis of other secondary metabolites", "carbohydrate metabolism", "energy metabolism", "glycan biosynthesis and metabolism", "lipid metabolism", and "metabolism of cofactors and vitamins". All KEGG annotations provided a valuable resource for investigating specific processes, functions, and pathways in the peony leaf.

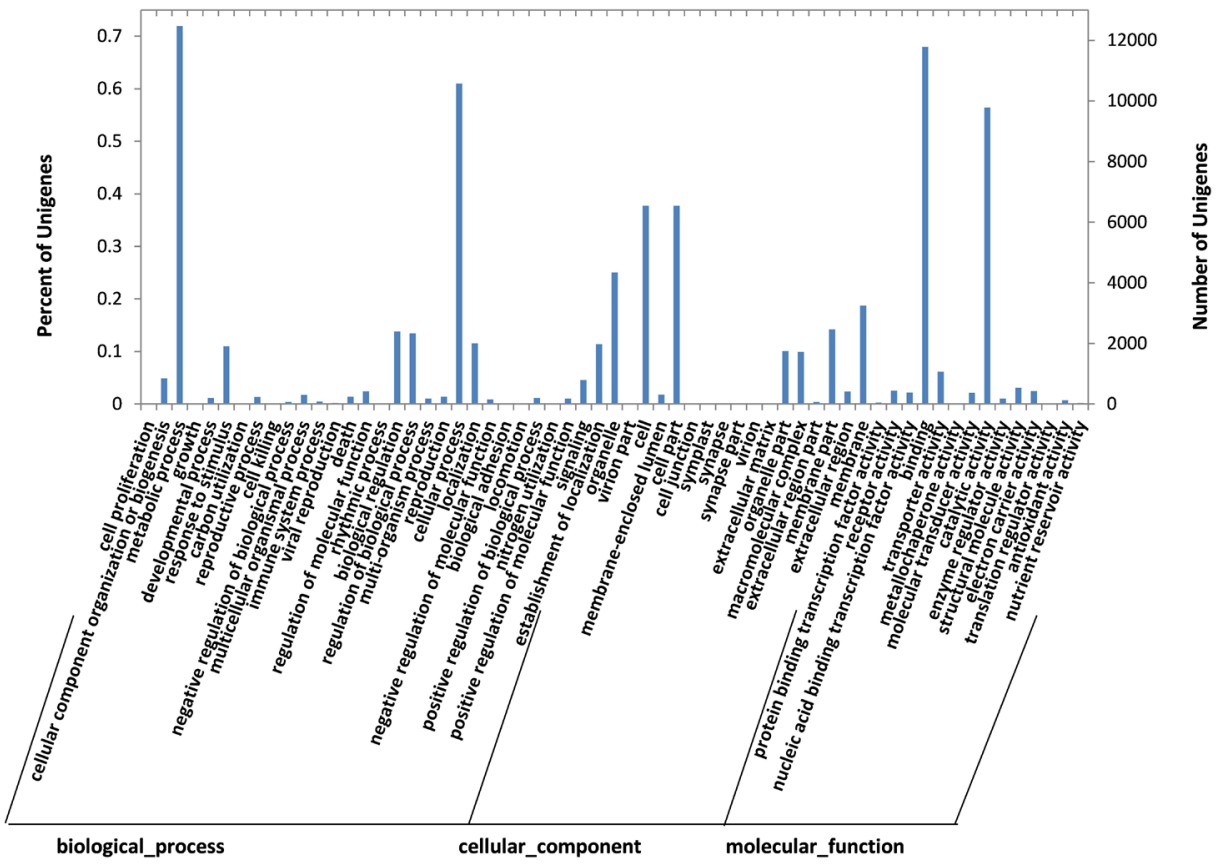

Figure 4. Histogram of clusters in KOG classification. All unigenes were aligned to the KOG database to predict and classify the possible function.

\section{Identification of $\mathrm{Hsps}$}

To enhance the thermal tolerance of tree peony, we sought to identify $H s p$ s in the transcriptomes of peony leaf. Through BLAST searching the $\mathrm{Nr}$ protein database with an E-value of 1e-5, a total of 64 Hsps were identified (Table S1). Of these Hsps, 24 members contained a complete open reading frame with start and stop codons and 40 members corresponding to partial sequences. The complete $H s p$ candidates were renamed as shown in Table 1, and the complete Hsp sequences are shown in Table S2. Phylogenetic analysis of the complete Hsps combined with gene annotation resulted in 5 members segregated into the Hsp90 family, 5 members into the Hsp70s family, and 14 members in the sHsp family (Figure 6). For sHsp groups, the PsHsp10.5-1, PsHsp10.5-2, PsHsp13.6, and PsHsp20.6 could not be closely grouped with other sHsp members. This is consistent with previous studies that sHsps originated from multiple genes families with much lower sequence similarity (Vierling, 1991; Waters et al., 1996; Wang et al., 2004). 

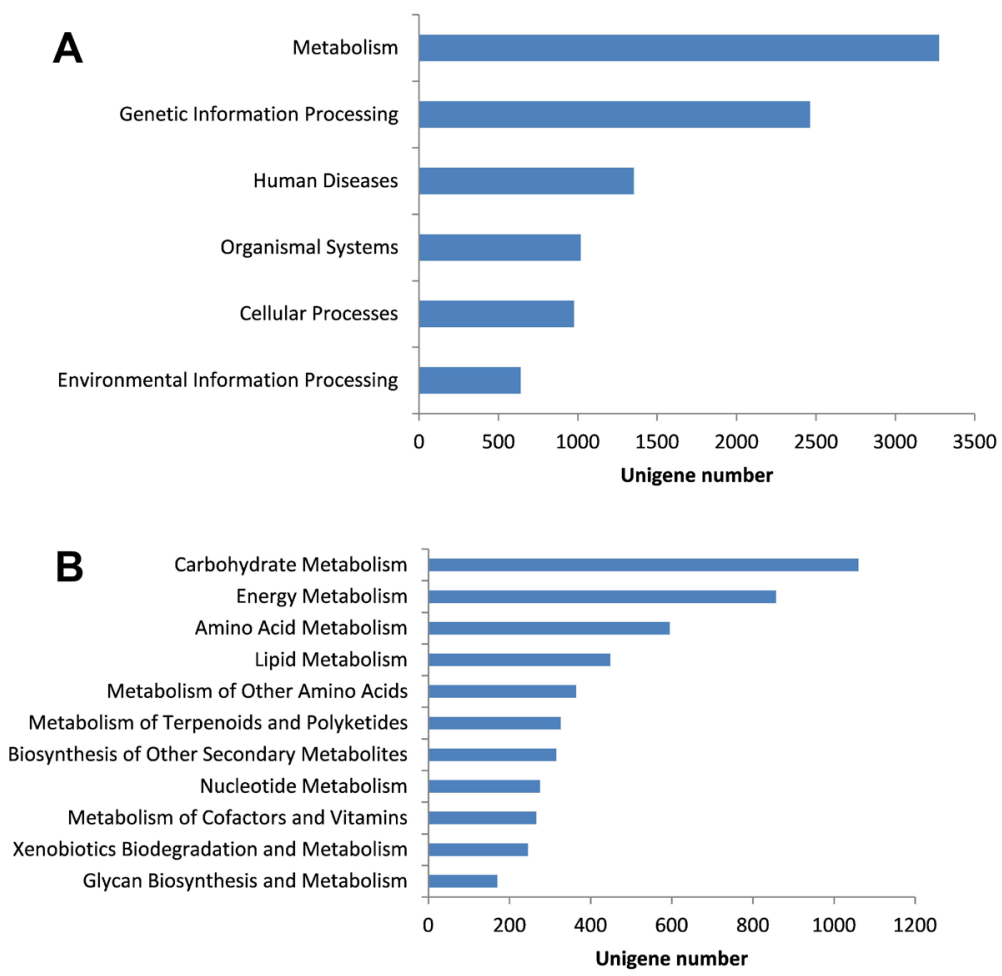

Figure 5. Pathway assignment based on KEGG. A. The main categories of KEGG pathway. B. Classification based on metabolism categories.

Table 1. Tree peony $H s p$ genes with complete open reading frames (ORFs).

\begin{tabular}{llll}
\hline Renamed gene & Unigene & Hsp family & Predicted location \\
\hline PsHsp90-1 & comp75883_c0_seq1 & Hsp90 & Endoplasmic reticulum. Mitochondrion \\
PsHsp90-2 & comp78211_c0_seq1 & Hsp90 & Endoplasmic reticulum \\
PsHsp90-3 & comp77605_c0_seq1 & Hsp90 & Cytoplasm \\
PsHsp90-4 & comp50451_c0_seq1 & Hsp90 & Cytoplasm \\
PsHsp90-5 & comp78749_c0_seq2 & Hsp90 & Cytoplasm \\
PsHsp70-1 & comp69431_c0_seq1 & Hsp70 & Endoplasmic reticulum. Mitochondrion \\
PsHsp70-2 & comp65891_c0_seq1 & Hsp70 & Mitochondrion \\
PsHsp70-3 & comp58928_c__seq1 & Hsp70 & Mitochondrion \\
PsHsp70-4 & comp77635_c0_seq1 & Hsp70 & Mitochondrion \\
PsHsp70-5 & comp50556_c0_seq1 & Hsp70 & Mitochondrion \\
PsHsp10.5-1 & comp70063_c0_seq2 & sHsp & Chloroplast \\
PsHsp10.5-2 & comp327433_c0_seq1 & sHsp & Mitochondrion. Nucleus \\
PsHsp12.4 & comp56933_c0_seq1 & sHsp & Nucleus \\
PsHsp13.6 & comp59750_c0_seq1 & sHsp & Golgi apparatus. Nucleus \\
PsHsp15.6 & comp46119_c0_seq1 & sHsp & Chloroplast \\
PsHsp16.4 & comp75870_c1_seq12 & sHsp & Chloroplast. Cytoplasm \\
PsHsp17.5 & comp75014_c0_seq2 & sHsp & Chloroplast. Cytoplasm \\
PsHsp17.6 & comp117581_c0_seq1 & sHsp & Chloroplast. Cytoplasm \\
PsHsp18.1 & comp77828_c0_seq25 & sHsp & Nucleus \\
PsHsp18.2 & comp71824_c0_seq2 & sHsp & Nucleus \\
PsHsp20.6 & comp78228_c0_seq1 & sHsp & Chloroplast \\
PsHsp23.3 & comp62722_c1_seq2 & sHsp & Chloroplast \\
PsHsp25.9-1 & comp65352_c0_seq1 & sHsp & Chloroplast \\
PsHsp25.9-2 & comp64630_c0_seq1 & sHsp & Chloroplast \\
\hline & & &
\end{tabular}




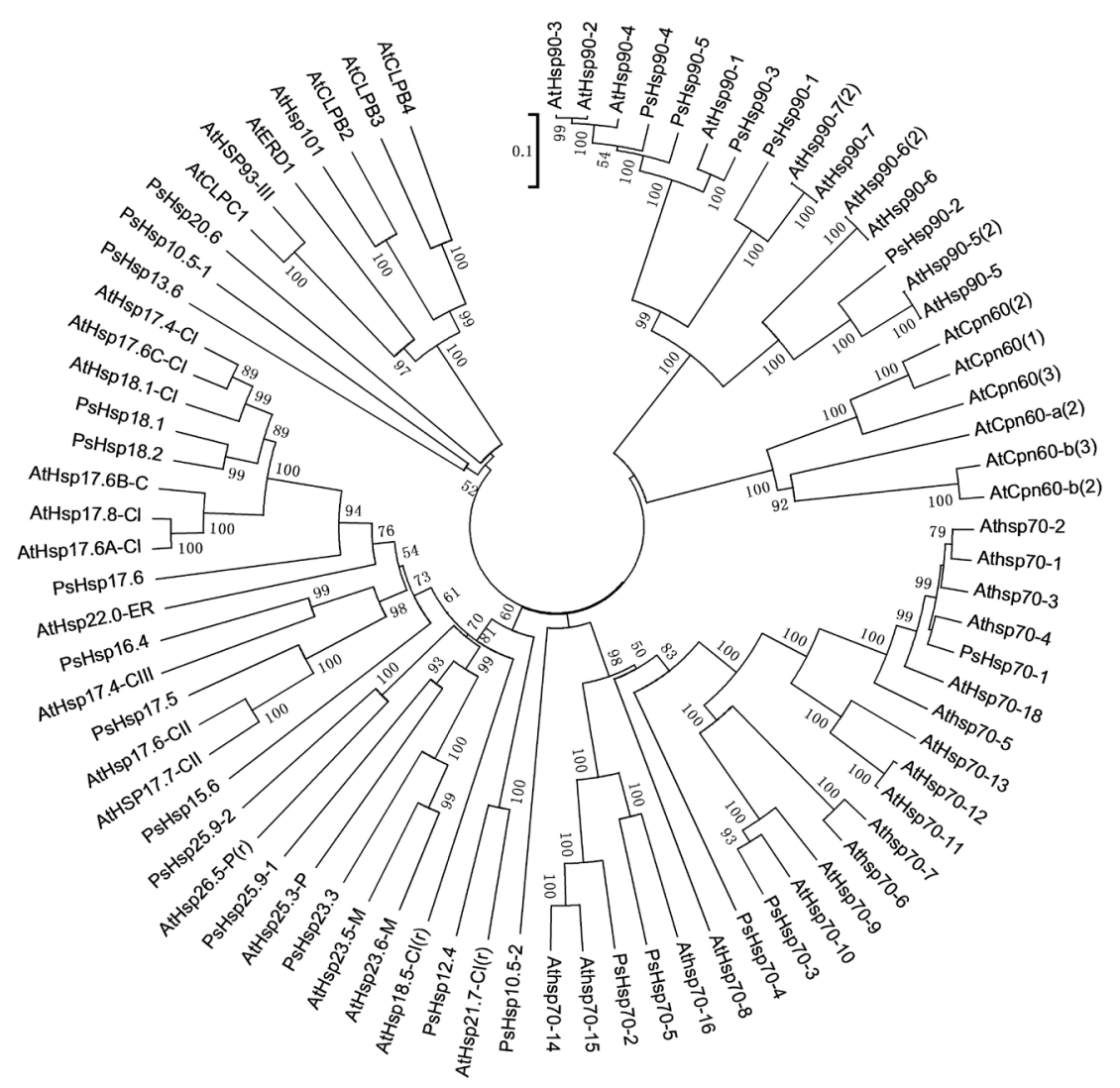

Figure 6. Phylogenetic analysis of the Hsps from tree peony and Arabidopsis. Complete sequences were aligned using ClustalW with default settings. The phylogenetic tree was constructed using MEGA5 with the neighborjoining method. Percentage bootstrap support values above $50 \%$ based on 1000 replicates are shown.

Hsp90 proteins are essential and highly conserved molecular chaperones in plants. They have multiple functions and play a major role in managing proteins folding (Buchner, 1999; Richter and Buchner, 2001). The expression of $H s p 90$ is elevated in response to environmental stressors such as heat, cold, salt, and heavy metal exposure (Milioni and Hatzopoulos, 1997; Krishna and Gloor, 2001). In Arabidopsis, 7 Hsp90 members have been identified and analyzed. The members from AtHsp90-1 through AtHsp90-4 were predicted to be located in cytoplasmic areas, while the other 3 members (AtHsp90-5, AtHsp90-6, and AtHsp90-7) were predicted to be localized in plastids, mitochondria, and the endoplasmic reticulum, respectively (Krishna and Gloor, 2001). In this study, sequence identity ranged from 43 to $92 \%$ among PsHsp70 members and from 42 to 93\% with orthologous Hsp90 from Arabidopsis. Intracellular localization prediction revealed that PsHsp90-1 was located in the endoplasmic reticula and mitochondria; PsHsp90-2 was located in the endoplasmic reticula; and PsHsp90-3, PsHsp90-4, and PsHsp90-5 were located in the cytoplasm (Table 1).

Hsp70 members are also conserved and represent stress-tolerance proteins. In Arabidopsis, the Hsp70 family contains 18 members divided into the DnaK subfamily (14 members) 
and Hsp110/SSE subfamily (4 members). Members of the DnaK subfamily were assigned to distinct subcellular locations, including the plastid, cytoplasm, endoplasmic reticulum, and mitochondrion (Lin et al., 2001). Sequence identities ranged from 18 to 54\% among PsHsp70 members and from 20 to $93 \%$ with orthologous Hsp70 from Arabidopsis. Location prediction revealed that PsHsp70-1 was located in the endoplasmic reticula and mitochondria, while the other 4 Hsp70 members (PsHsp70-2 through PsHsp70-5) were located in the mitochondria (Table 1). sHsps have molecular weights ranging from 10 to $40 \mathrm{kDa}$ and form more diverse subfamilies than Hsp90s and Hsp70s based on sequence identity. In higher plants, 6 gene families encoding sHsps have been identified. These families were found to be located in different cellular components including chloroplasts, endoplasmic reticula, cytosols, and mitochondria (Lenne and Douce, 1994; Waters et al., 1996). The sHsps are thought to be important for thermal stress responses. Most of the sHsps were not detected in normal vegetative tissues, but showed high accumulation when the plant was subjected to heat shock stress (Sun et al., 2002). The sHsps mainly function by binding to non-native proteins to stabilize and prevent non-native aggregation, which facilitates the subsequent refolding by ATP-dependent chaperones (Lee et al., 1997; Lee and Vierling, 2000). In Arabidopsis, 13 sHsp members were identified and divided into 6 subgroups based on sequence relatedness and intracellular localization. In our study, the sequence identities of the PssHsp members ranged from 3 to $85 \%$, while identities ranged from 3 to $82 \%$ with orthologous sequences from Arabidopsis. These data indicate that sHsps have poor sequence conservation. Location prediction revealed that four members were located in chloroplasts, 3 members in both chloroplasts and cytoplasm, 3 members in nuclei, 1 in Golgi apparatus and nuclei, and 1 in mitochondria and nuclei (Table 1).

\section{DISCUSSION}

Transcriptome resources for peony leaf are not yet available. In this study, transcriptomes of tree peony leaf exposed to high temperatures were sequenced using the HiSeq 2000 platform. We assembled 93,714 unigenes with an average length of $639.7 \mathrm{bp}$. Two previous studies reported the transcriptome sequencing of tree peony petals and flower buds. Zhang et al. (2013) identified 50,829 unigenes with an average length of $585 \mathrm{bp}$. Zhou et al. (2013) generated 59,275 and 63,962 unigenes with mean sizes of 698 and $699 \mathrm{bp}$ from 2 types of tree peonies, respectively. Compared with these 2 studies, we obtained a larger number of unigenes in the leaf transcriptome with longer or similar average lengths. These data indicate that high-quality reads were obtained from Illumina sequencing and effectively assembled and that the transcriptome sequences obtained in this study increased the transcriptome nucleotide coverage in tree peony.

Since a reference genome and leaf transcriptome for tree peony-related species are lacking, it was difficult to estimate potential functions using de novo assembly. Therefore, we evaluated the unigenes by blasting against public databases. A total of $23.8 \%$ of the unigenes had homologs in the $\mathrm{Nr}$ database. The matching of our unigenes in public databases indicated that the Illumina sequencing yielded a substantial fraction of unique genes from the leaf transcriptome of tree peony. However, compared with the transcriptomes from rubber tree (Li et al., 2012), carrot (Iorizzo et al., 2011), and lodgepole pine (Parchman et al., 2010), more unigenes from our sequencing project were not matched with unique proteins in public databases. Most large-scale sequencing studies on tree peony have focused on the floral organ, and transcriptomic information of leaves in peony-related species has not been included in public 
databases prior to this study. Therefore, we suggest that many of the unigenes without database hits may be novel genes discovered in tree peony. However, other factors may have also led to the failure of homology matching. For instance, shorter sequences may lack a characterized protein domain or the sequences may belong to untranslated regions.

The ability to withstand thermal stress is very important in plant development. Heat stress can trigger a number of cellular responses to minimize harm, the most predominant of which is elevated expression of Hsps. In plants, Hsps have been divided into the Hsp100, Hsp90, Hsp70, Hsp60, and sHsp families. Individual members of each class of Hsps have particular functions, but they may coordinate or synergistically interact with others stress-response mechanisms to prevent cellular damage and reestablish cellular homeostasis (Rossel et al., 2002; Wang et al., 2004). Hsps have been used as candidate genes for breeding thermal tolerance in plants, such as Hsp101 in Arabidopsis (Murakami et al., 2004), Hsp17.7 in rice (Sato and Yokoya, 2008), and CpsHsp in tomato (Wang et al., 2005). In this study, we identified 24 putative Hsps, including 5 members of Hsp90, 5 members of Hsp70, and 14 members of sHsp. The predicated Hsps in each group were relatively conserved and showed sequence similarity with their homologous protein in Arabidopsis. The newly identified Hsps will increase the understanding of how the tree peony responds to the environment and can also be exploited as novel genes for molecular breeding of tree peony-related species with improved thermal tolerance.

\section{ACKNOWLEDGMENTS}

Research supported by the NSFC-Henan Province Joint Foundation (\#U1204307), the NSFC foundation (\#31400602), and the Cultivation Foundation supported by Luoyang Normal University (\#2013-PYJJ-001). We also would like to acknowledge the Encode Genomics BioTechnology Co., Ltd. (Suzhou, China) for assistance with transcriptome sequencing and the related bioinformatic analysis.

\section{Supplementary material}

\section{REFERENCES}

Agarwal M, Katiyar-Agarwal S, Sahi C, Gallie DR, et al. (2001). Arabidopsis thaliana Hsp100 proteins: kith and kin. Cell Stress Chaperones 6: 219-224.

Buchner J (1999). Hsp90 \& Co. - a holding for folding. Trends Biochem. Sci. 24: 136-141.

Fu L, Niu B, Zhu Z, Wu S, et al. (2012). CD-HIT: accelerated for clustering the next-generation sequencing data. Bioinformatics 28: 3150-3152.

Grabherr MG, Haas BJ, Yassour M, Levin JZ, et al. (2011). Full-length transcriptome assembly from RNA-Seq data without a reference genome. Nature Biotechnol. 29: 644-652.

Hill JE and Hemmingsen SM (2001). Arabidopsis thaliana type I and II chaperonins. Cell Stress Chaperones 6: 190-200.

Iorizzo M, Senalik DA, Grzebelus D, Bowman M, et al. (2011). De novo assembly and characterization of the carrot transcriptome reveals novel genes, new markers, and genetic diversity. BMC Genomics 12: 389.

Katiyar-Agarwal S, Agarwal M and Grover A (2003). Heat-tolerant basmati rice engineered by over-expression of hsp 101. Plant Mol. Biol. 51: 677-686.

Krishna P and Gloor G (2001). The Hsp90 family of proteins in Arabidopsis thaliana. Cell Stress Chaperones 6: 238-246.

Kumar RR, Goswami S, Sharma SK, Singh K, et al. (2012). Protection against heat stress in wheat involves change in cell membrane stability, antioxidant enzymes, osmolyte, $\mathrm{H}_{2} \mathrm{O}_{2}$ and transcript of heat shock protein. Int. J. Plant Physiol. Biochem. 4: 83-91.

Lee GJ and Vierling E (2000). A small heat shock protein cooperates with heat shock protein 70 systems to reactivate a heat-denatured protein. Plant Physiol. 122: 189-198. 
Lee GJ, Roseman AM, Saibil HR and Vierling E (1997). A small heat shock protein stably binds heat-denatured model substrates and can maintain a substrate in a folding-competent state. EMBO J. 16: 659-671.

Lenne C and Douce R (1994). A low molecular mass heat-shock protein is localized to higher plant mitochondria. Plant Physiol. 105: 1255-1261.

Li C, Zhao D and Tao J (2011). Molecular cloning and sequence analysis of a heat shock protein gene PsHSP70 from tree peony (Paeonia suffruticosa Andr.). J. Yangzhou Univ. (Agric. Life Sci. Edn.) 32: 55-58.

Li D, Deng Z, Qin B, Liu X, et al. (2012). De novo assembly and characterization of bark transcriptome using Illumina sequencing and development of EST-SSR markers in rubber tree (Hevea brasiliensis Muell. Arg.). BMC Genomics 13: 192.

Lin BL, Wang JS, Liu HC, Chen RW, et al. (2001). Genomic analysis of the Hsp70 superfamily in Arabidopsis thaliana. Cell Stress Chaperones 6: 201-208.

Maestri E, Klueva N, Perrotta C, Gulli M, et al. (2002). Molecular genetics of heat tolerance and heat shock proteins in cereals. Plant Mol. Biol. 48: 667-681.

Meyer E, Aglyamova G, Wang S, Buchanan-Carter J, et al. (2009). Sequencing and de novo analysis of a coral larval transcriptome using 454 GSFlx. BMC Genomics 10: 219.

Milioni D and Hatzopoulos P (1997). Genomic organization of hsp90 gene family in Arabidopsis. Plant Mol. Biol. 35: 955-961.

Murakami T, Matsuba S, Funatsuki H, Kawaguchi K, et al. (2004). Over-expression of a small heat shock protein, sHSP17. 7, confers both heat tolerance and UV-B resistance to rice plants. Mol. Breed. 13: 165-175.

Parchman T, Geist K, Grahnen J, Benkman C, et al. (2010). Transcriptome sequencing in an ecologically important tree species: assembly, annotation, and marker discovery. BMC Genomics 11: 180.

Queitsch C, Hong SW, Vierling E and Lindquist S (2000). Heat shock protein 101 plays a crucial role in thermotolerance in Arabidopsis. Plant Cell 12: 479-492.

Richter K and Buchner J (2001). Hsp90: chaperoning signal transduction. J. Cell. Physiol. 188: 281-290.

Rossel JB, Wilson IW and Pogson BJ (2002). Global changes in gene expression in response to high light in Arabidopsis. Plant Physiol. 130: 1109-1120.

Sato Y and Yokoya S (2008). Enhanced tolerance to drought stress in transgenic rice plants overexpressing a small heatshock protein, sHSP17.7. Plant Cell Rep. 27: 329-334.

Scharf KD, Siddique M and Vierling E (2001). The expanding family of Arabidopsis thaliana small heat stress proteins and a new family of proteins containing $\alpha$-crystallin domains (Acd proteins). Cell Stress Chaperones 6: 225-237.

Sun L, Liu Y, Kong X, Zhang D, et al. (2012). ZmHSP16. 9, a cytosolic class I small heat shock protein in maize (Zea mays), confers heat tolerance in transgenic tobacco. Plant Cell Rep. 31: 1473-1484.

Sun W, Van Montagu M and Verbruggen N (2002). Small heat shock proteins and stress tolerance in plants. Biochim. Biophys. Acta 1577: 1-9.

Tamura K, Peterson D, Peterson N, Stecher G, et al. (2011). MEGA5: molecular evolutionary genetics analysis using maximum likelihood, evolutionary distance, and maximum parsimony methods. Mol. Biol. Evol. 28: 2731-2739.

Toledo-Silva G, Cardoso-Silva CB, Jank L and Souza AP (2013). De novo transcriptome assembly for the tropical grass Panicum maximum Jacq. PloS One 8: e70781.

Vera JC, Wheat CW, Fescemyer HW, Frilander MJ, et al. (2008). Rapid transcriptome characterization for a nonmodel organism using 454 pyrosequencing. Mol. Ecol. 17: 1636-1647.

Vierling E (1991). The roles of heat shock proteins in plants. Annu. Rev. Plant Biol. 42: 579-620.

Wang L, Zhao CM, Wang YJ and Liu J (2005). Overexpression of chloroplast-localized small molecular heat-shock protein enhances chilling tolerance in tomato plant. Zhi Wu Sheng Li Yun Fen Sheng Wu Xue Xue Bao 31: 167-174.

Wang W, Vinocur B, Shoseyov O and Altman A (2004). Role of plant heat-shock proteins and molecular chaperones in the abiotic stress response. Trends Plant Sci. 9: 244-252.

Waters ER, Lee GJ and Vierling E (1996). Evolution, structure and function of the small heat shock proteins in plants. $J$. Exp. Bot. 47: 325-338.

Xue GP, Sadat S, Drenth J and Mcintyre CL (2014). The heat shock factor family from Triticum aestivum in response to heat and other major abiotic stresses and their role in regulation of heat shock protein genes. J. Exp. Bot. 65: 539-557.

Zhang C, Wang Y, Fu J, Dong L, et al. (2013). Transcriptomic analysis of cut tree peony with glucose supply using the RNA-Seq technique. Plant Cell Rep. 33: 111-129.

Zhou H, Cheng FY, Wang R, Zhong Y, et al. (2013). Transcriptome comparison reveals key candidate genes responsible for the unusual reblooming trait in tree peonies. PloS One 8: e79996. 